\title{
Efectos psicológicos de la práctica del Método Pilates en una muestra universitaria
}

\author{
Eva Herrera-Gutiérrez*, Marina Olmos-Soria y David Brocal-Pérez
}

Departamento de Psicología Evolutiva y de la Educación. Facultad de Psicología. Universidad de Murcia (España)

\begin{abstract}
Resumen: El propósito general de esta investigación fue comprobar los posibles efectos psicológicos de la práctica del Método Pilates y, en particular, las consecuencias sobre el grado de ansiedad experimentado. Éste es un método de entrenamiento físico enfocado a la mejora de la higiene postural y del bienestar psicológico, altamente recomendado en la actualidad por los profesionales sanitarios, tanto para personas con patologías diagnosticadas como con fines preventivos. Hasta el momento se han comprobado los beneficios físicos y psicológicos asociados a la práctica regular de actividad física. Sin embargo, se han realizado pocos estudios sobre los efectos concretos del Método Pilates. En el presente trabajo se examinó la ansiedad (estado y rasgo) en un grupo sedentario y otro grupo practicante habitual de este método (estudiantes, personal y usuarios de las instalaciones deportivas de la Universidad de Murcia, España). Para evaluar la ansiedad se utilizó el Cuestionario de Ansiedad Estado-Rasgo (STAI) de Spielberger, Gorsuch y Lushene (2002). Se discuten las repercusiones que los resultados de este trabajo pueden tener en la promoción de la salud en el ámbito universitario y su contribución a la salud pública.

Palabras clave: promoción de la salud; Método Pilates; actividad física; deporte; efectos psicológicos; ansiedad; estudiantes; universidad.
\end{abstract}

Title: Psychological effects of the practice of Pilates Method in a university sample.

Abstract: The overall purpose of this research was to test the possible psychological effects of the practice of Pilates and, in particular, the effect on the degree of anxiety experienced. This is a method of physical training aimed at improving postural hygiene and psychological well-being, highly recommended today by health professionals, both for people with diagnosed diseases and for preventive level. So far, the physical and psychological benefits associated with regular physical activity practice have already been demonstrated. However, only a few studies have investigated the specific effects of the Pilates Method. This paper examined the anxiety (state and trait) in a sedentary group and in another group of usual practitioners of this method (staff, students, and users of sports facilities at the University of Murcia, Spain). The State-Trait Anxiety Inventory (STAI) of Spielberger, Gorsuch and Lushene (2002) was used to assess anxiety. The implications that the results of this work can have from the perspective of health promotion in the university are discussed, as well as its contribution to public health.

Key words: health promotion; Pilates Method; physical activity; sport; psychological effects; anxiety; students; university.

\section{Introducción}

Cuando abordamos el tema de Salud Pública, uno de los objetivos prioritarios es la promoción de la salud, entendida no como la ausencia de enfermedad, sino como un estado de bienestar físico, mental y social, tal y como actualmente la define la OMS.

Entre las medidas de promoción de la salud, y en un intento de reducir los principales factores de enfermedad y mortalidad (como las enfermedades cardiovasculares), se aconseja la práctica de actividades físicas acordes a la edad de la persona y a su condición física y estado de salud (Tremblay, Colley, Saunders, Healy y Owen, 2010). No obstante, estamos en una sociedad cada vez más sedentaria, con un incremento en los niveles de obesidad y alteraciones cardiovasculares. Asimismo, el índice de trastornos mentales causados por el estrés y los problemas derivados de la vida cotidiana, tales como los propios trastornos de ansiedad o la depresión, va en aumento.

Implicar a las personas sedentarias en la práctica de actividades de carácter físico y, sobre todo, mantenerlas en el esfuerzo hasta lograr beneficios percibidos no es tarea fácil. A menudo se inicia algún tipo de actividad física que se abandona a las pocas semanas, cuando las responsabilidades hacen acusar la falta de tiempo. Aunque también existen diferencias psicofisiológicas y de personalidad entre personas deportistas y sedentarias (Herrera y Gómez-Amor, 1995,

* Dirección para correspondencia [Correspondence address]:

Eva Herrera-Gutiérrez. Departamento de Psicología Evolutiva y de la Educación. Facultad de Psicología. Campus Universitario de Espinardo. Universidad de Murcia. 30100 Murcia (España). E-mail: evahg@um.es
1996), por lo que en la inclinación hacia el ejercicio físico estarían involucrados determinados rasgos psicológicos.

En cualquier caso, lo cierto es que disponemos de una amplia literatura que avala los beneficios de la práctica frecuente de actividad física en poblaciones muy diferentes. Numerosos estudios han demostrado que la práctica habitual del ejercicio físico se relaciona directamente con una mejor salud psicológica y calidad de vida, tanto en niños y jóvenes (Herrera-Gutiérrez, Brocal-Pérez, Sánchez y Rodríguez, 2012; Tubic y Djordjic, 2013) como en personas mayores (Coll et al., 2009).

Así, se han observado mejoras en el bienestar somático y psicológico - en concreto, una disminución de los niveles de ansiedad y depresión- asociados a la práctica del deporte y del ejercicio físico (Asztalos, De Bourdeauhuij y Cardon, 2010; Ferney, Gilson, Brown, Burton y McKenna, 2009; Fitzsimons et al., 2008; Herrera, Gómez-Amor, Zamora y Sastre, 1993; Herrera Gutiérrez, 1994; Kull, 2003). Aspectos tales como el tipo, intensidad, duración y frecuencia de la actividad física son de especial importancia a la hora de producir algún beneficio psicológico (Kim et al., 2004; Wankel y Berger, 1990). Al parecer, los mejores beneficios se obtienen cuando se trata de una actividad física de intensidad moderada (Herrera Gutiérrez, 1994).

Actualmente, es frecuente la recomendación de la realización de ejercicio aeróbico (caminar, natación, ciclismo) o anaeróbico (yoga) de baja o media intensidad. Una de las actividades físicas que recientemente está ganando popularidad es el conocido como "Método Pilates", que goza de gran aceptación entre la población, sobre todo mujeres, por la rápida mejora que suele conllevar y la consecuente sensación de bienestar. 
Este método fue diseñado por Joseph Hubertus Pilates, a principios del siglo XX, en un intento de mejorar las condiciones físicas y mentales de pacientes en rehabilitación durante la I Guerra Mundial. Se trata de un programa de fitness mente-cuerpo basado en seis puntos clave: centración, concentración, control, precisión, respiración y flujo. Su objetivo es, fundamentalmente, mejorar el bienestar físico y mental a través de la coordinación de movimientos. El método incorpora el uso de rutinas de movimiento encaminadas a mejorar la flexibilidad, la fuerza y la coordinación. Se realiza mediante un programa de ejercicios en máquinas o en suelo acompañados en ocasiones de material complementario (balones, aros, cintas, etc.). Son ejercicios de coordinación inter e intramuscular enfocados a la mejora de la higiene postural, mediante el fortalecimiento y estiramiento de toda la musculatura, especialmente aquella que envuelve la columna vertebral.

Como decíamos anteriormente, es un método que se ha extendido rápidamente en las últimas décadas y cuenta con un amplio número de practicantes. Algunos estudios científicos han avalado su utilidad para la mejora de la fuerza y la resistencia de los músculos abdominales y lumbares, la flexibilidad del tronco posterior (Critchley, Pierson y Battersby, 2011; Sekendiz, Altun, Korkusuz y Akin, 2007), el equilibrio estático (Guedes, Cader, Valim, Oliveira y Martin, 2010) y dinámico (Jonson, Larsen, Ozawa, Wilson y Kennedy, 2007), la flexibilidad general (Segal, Hein y Basford, 2004) y la postura (Emery, De Serres, McMillan y Côté, 2010).

No obstante, escasean los estudios que aborden sus efectos psicológicos (Tinoco y Jiménez, 2010). Es conocido que el practicante habitual de Pilates tiene un perfil concreto: mujer de mediana edad que no suele practicar otra actividad física y que presenta dolor músculo-esquelético (Sperling y
Brum, 2006). Otras personas se acercan a este método como una forma de divertirse, salir de casa, socializarse, etc., es decir, buscando más los beneficios psicológicos y sociales que físicos. Llegados a este punto, cabría preguntarse: ¿es efectivo el Método Pilates para producir una mejora en el bienestar psicológico? Hasta el momento, tenemos poca información fehaciente a este respecto, aunque alguna investigación apunta en este sentido (por ejemplo, Caldwell, Harrison, Adams y Triplett, 2009).

Partiendo de estas premisas, el objetivo de este estudio fue examinar los efectos psicológicos de la práctica del Método Pilates y, en concreto, las consecuencias sobre el nivel de ansiedad experimentado. Este trabajo forma parte de una investigación más amplia encaminada a conocer las relaciones entre la práctica de dicho método y el bienestar psicológico.

\section{Método}

\section{Participantes}

La muestra estuvo compuesta por 47 jóvenes, de edades comprendidas entre los 18 y los 31 años $(M=23.47$; $D T=$ 3.95). Por género, había 5 varones y 42 mujeres. Todos fueron seleccionados en el contexto de la Universidad de Murcia, España. De ellos, 24 eran practicantes de Pilates y 23 no practicantes de Pilates ni de ninguna otra actividad física. Alrededor del $80 \%$ de los practicantes solía realizar 2 sesiones semanales de 1 hora de Pilates en suelo, el resto variaba entre 1 y 7 sesiones. La Figura 1 muestra la distribución por horas semanales de práctica de los sujetos.

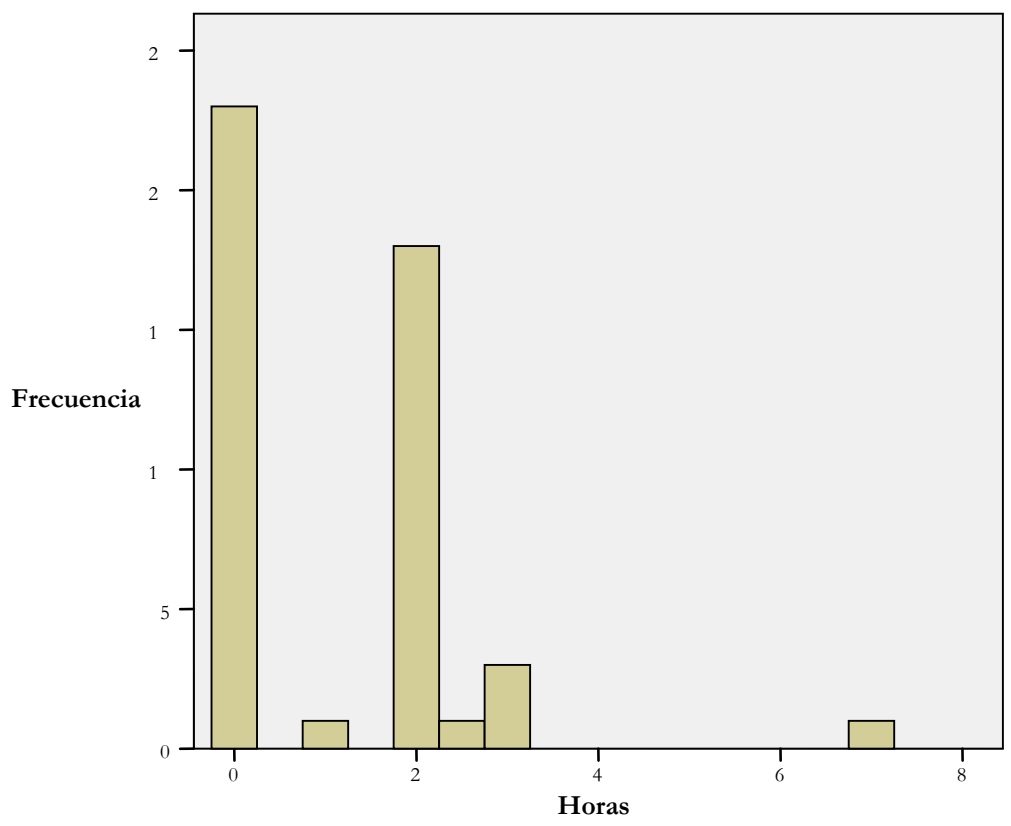

Figura 1. Horas de práctica de Pilates por semana. 
En cuanto a los meses de práctica, ésta variaba entre 1 y 24 meses. De los datos disponibles, 7 sujetos habían practi- cado durante menos de un año y 4 más de un año. En la Figura 2 se presentan las frecuencias para esta variable.

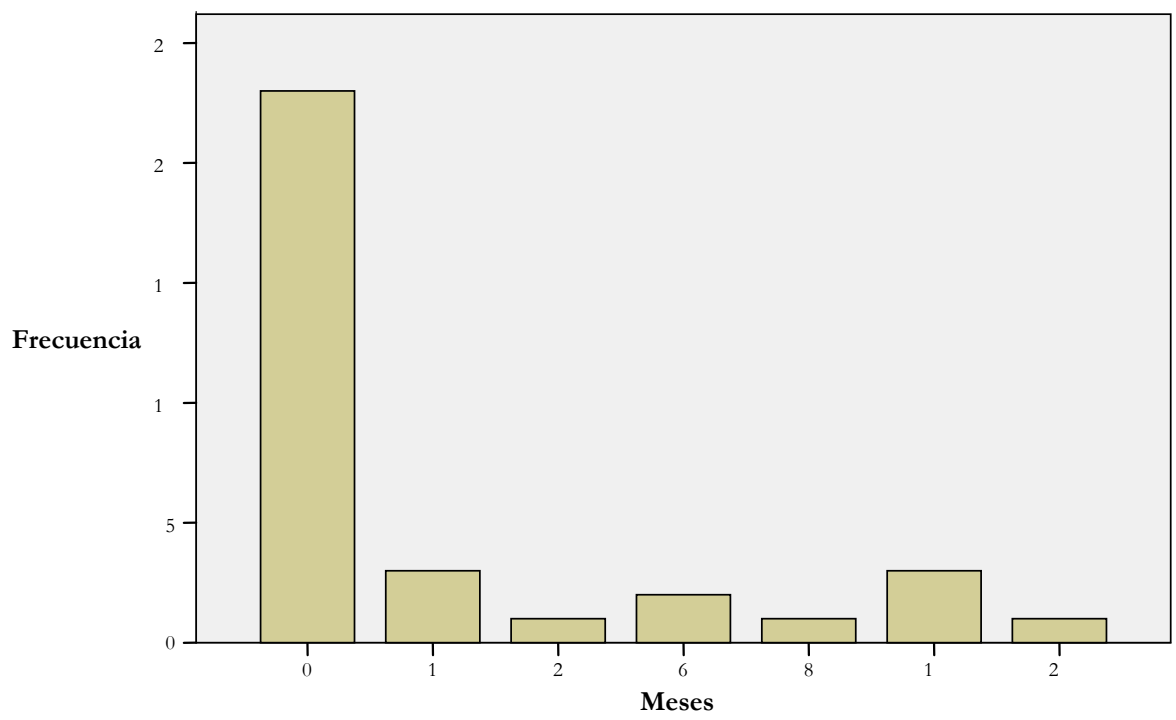

Figura 2. Meses de práctica de Pilates.

\section{Instrumentos}

A todos los sujetos se les administró el Cuestionario de Ansiedad Estado-Rasgo (STAI; Spielberger, Gorsuch y Lushene, 2002) más un cuestionario sobre datos personales y práctica de Pilates y otras actividades físicas que pudieran realizar.

\section{Procedimiento}

Se seleccionaron los sujetos entre los alumnos de la Universidad de Murcia que o bien no realizaban ninguna actividad física o bien eran usuarios de los cursos de Pilates que esta institución ofrece. Se repartieron los cuestionarios entre los sujetos y los fueron devolviendo en el plazo de una semana.

\section{Análisis de datos}

Se obtuvieron estadísticos descriptivos básicos (medias, desviaciones típicas, frecuencias y porcentajes) para cada una de las variables consideradas en este estudio. Para comparar la ansiedad estado y rasgo de los dos grupos de universitarios se aplicó la prueba $t$ de significación de diferencias entre medias de muestras independientes. El supuesto de homogeneidad de varianzas se evaluó con la prueba de Levene. Se estimó el tamaño del efecto mediante el índice 'diferencias de medias estandarizada' (Cohen, 1988). Debido al bajo tamaño muestral, se calculó la potencia a posteriori de los contrastes realizados. También se llevó a cabo un análisis de co- rrelaciones para observar las pautas de variación común entre las puntuaciones en las escalas de ansiedad y el número de horas semanales de práctica, así como el de meses practicando el deporte.

El tratamiento estadístico de los datos se hizo con el paquete de software estadístico SPSS, versión 15.0. El nivel de significación estadística asumido fue del 5\% ( $p \leq .05)$.

\section{Resultados}

La Tabla 1 presenta los estadísticos descriptivos obtenidos por los dos grupos de participantes en las variables ansiedadestado y ansiedad-rasgo. En personas practicantes de Pilates las puntuaciones medias en ambas variables fueron similares. En cambio, en personas sedentarias la ansiedad-rasgo fue ligeramente más alta, siendo la ansiedad-estado muy semejante a la de los practicantes. Las dos pruebas de Levene resultaron ser estadísticamente no significativas $(p=.943$ у $p=$ .242 para ansiedad-estado y ansiedad-rasgo, respectivamente), indicando que se cumplió el supuesto de homogeneidad de las varianzas.

Tabla 1. Estadísticos descriptivos de Ansiedad Estado (STAIE) y Rasgo (STAIR) en practicantes de Pilates y sedentarios.

\begin{tabular}{lcccccc}
\hline & \multicolumn{3}{c}{ Grupo Pilates } & \multicolumn{3}{c}{ Grupo Sedentarios } \\
\cline { 2 - 7 } Variable & $N$ & Media & $D T$ & $n$ & Media & $D T$ \\
\hline STAI-E & 24 & 16.88 & 10.50 & 23 & 16.61 & 0.70 \\
STAI-R & 24 & 16.88 & 11.69 & 23 & 21.96 & 8.39 \\
\hline
\end{tabular}

$n$ : tamaño muestral. DT: desviación típica. STAI-E: ansiedad-estado. STAI-

R: ansiedad-rasgo. 
La prueba $t$ de comparación de medias no arrojó diferencias estadísticamente significativas entre las personas practicantes y no practicantes en las variables ansiedadestado $[t(45)=0.089, p=.929]$ y ansiedad-rasgo $[t(45)=$ $1.706, p=.095]$. Para ansiedad-estado, el tamaño del efecto, en términos de la diferencia de medias estandarizada, fue $d=$ 0.02 , mientras que para ansiedad-rasgo fue $d=0.50$. Siguiendo el criterio de Cohen (1988), según el cual valores $d$ en torno a $0.2,0.5$ y 0.8 pueden considerarse reflejo de una magnitud del efecto baja, media y alta, respectivamente, cabe entender que no hubo efecto de la práctica del Método Pilates en ansiedad-estado. Sin embargo, en lo que respecta a la ansiedad-rasgo, el efecto obtenido es de magnitud media y, por ende, clínicamente relevante, en favor de dicho método. Para cada una de las pruebas $t$ aplicadas, la potencia estadística a posteriori fue de 0.051 y de 0.386 para ansiedad-estado y ansiedad-rasgo, respectivamente. Por tanto, la ausencia de significación en ansiedad-rasgo se debió a un problema de baja potencia estadística provocada por el reducido tamaño muestral.

Según las correlaciones de Pearson efectuadas (véase la Tabla 2), se obtuvo una asociación estadísticamente significativa de signo negativo entre las horas de práctica a la semana y la ansiedad-rasgo $(r=-.325, p<.05)$, y entre los meses de práctica y la ansiedad-rasgo $(r=-.476, p<.01)$. También se observó una relación marginalmente significativa $y$ negativa entre los meses de práctica de Pilates y la ansiedad-estado $(r=-.328, p=.058)$.

Tabla 2. Correlaciones entre la Ansiedad -Estado (STAIE) y Rasgo (STAIR) - y las variables actividad física (deporte), horas de práctica (horas) y meses de práctica (meses).

\begin{tabular}{lcc}
\hline Variable & Horas x semana & Meses de práctica \\
\hline Meses de práctica & $.688^{* *}$ & -- \\
STAI-E & $(n=34)$ & $-.328^{\mathrm{a}}$ \\
& -.096 & $(n=34)$ \\
STAI-R & $(n=47)$ & $-.476^{* *}$ \\
& $-.325^{*}$ & $(n=34)$ \\
\hline${ }^{*} p<.05 .{ }^{* *} p<.01 .{ }^{a} p=.058$. STAI-E: ansiedad-estado. STAI-R: ansie-
\end{tabular}
dad-rasgo.

\section{Discusión y conclusiones}

Estos resultados nos indican que las posibles diferencias en ansiedad que puedan aparecer entre personas practicantes del Método Pilates y sedentarias dependen en gran medida del tiempo y la intensidad de la práctica deportiva. Así, a mayor número de horas de práctica semanal y de meses de práctica, la ansiedad es menor.

Un resultado curioso fue el de una menor ansiedad rasgo en las personas deportistas en comparación con las sedentarias. También resulta digno de mención lo igualadas que están las medias en ansiedad-rasgo y en ansiedad-estado en el grupo de las personas deportistas. Los sedentarios, en cambio, presentaron mayores puntuaciones de ansiedad-rasgo, aunque en ansiedad-estado, en el momento del estudio, casi igualaban a los deportistas.

Este resultado apunta hacia la idea de que las personas naturalmente ansiosas quizás no busquen la actividad física como una forma de reducir su estrés. En este sentido, cabría incentivar desde los servicios deportivos universitarios la práctica del ejercicio físico como un factor generador de bienestar psicológico. Aunque también es posible que el perfil de persona que se acerca al Método Pilates sea alguien con una ansiedad-rasgo moderada. Esto lo puede corroborar el hecho de que en nuestro estudio la ansiedad rasgo es marcadamente menor en las personas que lo han practicado de forma habitual, durante largos periodos de tiempo.

En cuanto a la relación entre tiempo e intensidad de la práctica y ansiedad, era una relación esperada y acorde con la literatura, puesto que, como comentábamos en la introducción, se sabe que con niveles moderados de actividad física, la ansiedad disminuye (Herrera Gutiérrez, 1994).

Los resultados igualmente apuntan a que una práctica intensa del Método Pilates redunda en niveles todavía mayores de disminución de la ansiedad. Hay que considerar también que uno de los objetivos de este método es el bienestar mental, implicando ejercicios, como la concentración o la respiración, que benefician notablemente el afrontamiento de la ansiedad. Tales datos sugieren que, efectivamente, el Método Pilates, practicado con regularidad y durante tiempo prolongado, reduce de manera significativa los niveles de ansiedadestado.

Estos hallazgos complementan otros estudios sobre el bienestar psicológico asociado a la actividad física (Arruza et al., 2008; Asztalos et al., 2010; Ferney et al., 2009; HerreraGutiérrez et al., 2012) y apuntan hacia los posibles efectos beneficiosos de la práctica de este método en particular, en la línea de algunos de los escasos trabajos precedentes sobre el tema (Caldwell et al., 2009).

Es preciso señalar que la principal limitación de esta investigación es el escaso tamaño muestral disponible. Posiblemente, ésa puede haber sido la razón de no alcanzar diferencias estadísticamente significativas entre los dos grupos de participantes en la variable ansiedad-rasgo, a pesar de exhibir un tamaño del efecto relevante. Estudios futuros deberían intentar replicar estos resultados utilizando un tamaño muestral que garantice una potencia estadística apropiada.

No obstante, y a pesar de esta limitación, los resultados del presente estudio pueden tener implicaciones de interés desde el punto de vista de la promoción de la salud en el contexto universitario y su posible contribución a la salud pública. Cabría pues, recomendar la práctica del Método Pilates en la población universitaria, dados sus beneficios constatados en el ámbito psicológico. 


\section{Referencias}

Arruza, J. A., Arribas, S., Gil De Montes, L., Irazusta, S., Romero, S. y Cecchini, J. A. (2008). Repercusiones de la duración de la actividad físicodeportiva sobre el bienestar psicológico. Revista Internacional de Medicina y Ciencias de la Actividad Física y el Deporte, 8(30), 171-183.

Asztalos, M., De Bourdeaudhuij, I. y Cardon, G. (2010). The relationship between physical activity and mental health varies across activity intensity levels and dimensions of mental health among women and men. Public Health Nutrition, 13(8), 1207-1214. Disponible en: http://dx.doi.org/10.1017/S1368980009992825

Caldwell, K., Harrison, M., Adams, M. y Triplett, T. (2009). Effect of Pilates and taiji quan training on self-efficacy, sleep quality, mood and physical performance of college students. Journal of Bodywork and Movement Therapies, 13, 155-163. Disponible en: http://dx.doi.org/10.1016/i.jbmt.2007.12.001

Cohen, J. (1988). Statistical power analysis for the behavioral sciences ( $2^{\mathrm{a}}$ ed.). Hillsdale, NJ: Erlbaum.

Coll, G., Rodríguez, A., Vargas, S., Roig, L., Alsina, N., Comalada, C., Beltrán, M., Román, M., Planas, X. y Garre, J. (2009). Ejercicio físico y calidad de vida autopercibida en el anciano hipertenso. Hipertensión y Riesgo Cardiovascular, 26, 194-200. Disponible en: http://dx.doi.org/10.1016/i.hipert.2009.06.002

Critchley, D. J., Pierson, Z. y Battersby, G. (2011). Effect of pilates mat exercises and conventional exercise programmes on transversus abdominis and obliquus internus abdominis activity: Pilot randomised trial. Manual Therapy, 16, 183-189. Disponible en: http://dx.doi.org/10.1016/j.math.2010.10.007

Emery, K., De Serres, S.J., McMillan, A. y Côté, J. N. (2010). The effects of a Pilates training program on arm-trunk posture and movement. Clinical Biomechanics, 25, 124-130. Disponible en: http://dx.doi.org/10.1016/i.clinbiomech.2009.10.003

Ferney, S., Gilson, N., Brown, W., Burton, N. y McKenna, J. (2009). Walking experiences in an Australian university community: Qualitative perspectives with a randomised controlled trial. Journal of Science and Medicine in Sport, 12(S1), S78-S79. Disponible en: http://dx.doi.org/10.1016/i.jsams.2008.12.189

Fitzsimons, C. F., Baker, G., Wright, A., Nimmo, M. A., Ward, C., Lowry, R., Millington, C., Shaw, R., Fenwick, E., Ogilvie, D., Inchley, J., Foster, C. E. y Mutrie, N. (2008). The 'Walking for Wellbeing in the West' randomised controlled trial of a pedometer-based walking programme in combination with physical activity consultation with 12 month follow-up: rationale and study design. BMC Public Health, 8, 259-269. Disponible en: http://dx.doi.org/10.1186/1471-2458-8-259

Guedes, B., Cader, S., Valim, N., Oliveira, E. y Martin, E. H. (2010). Pilates method in personal autonomy, static balance and quality of life of elderly females. Journal of Bodywork and Movement Therapies, 14, 195-202. Disponible en: http://dx.doi.org/10.1016/j.jbmt.2009.12.005

Herrera, E. y Gómez-Amor, J. (1995). Differences in personality and menstrual variables between physically active and sedentary women. Personality and Individual Differences, 19(3), 389-392. Disponible en: http://dx.doi.org/10.1016/0191-8869(95)00071-D
Herrera, E. y Gómez-Amor, J. (1996). Diferencias psicofisiológicas y de personalidad entre mujeres deportistas y sedentarias. En E. Pérez Córdoba y J. C. Caracuel (Eds.), Psicología del deporte. Investigación y aplicación (pp. 155-159). Málaga: Instituto Andaluz del Deporte. Junta de Andalucía.

Herrera, E., Gómez-Amor, J., Zamora, S. y Sastre, J. (1993). Ejercicio físico y ciclo menstrual: Cambios hormonales, psicofisiológicos y sintomatología menstrual. Vigilia-Sueño, 3, 40.

Herrera Gutiérrez, E. (1994). Ejercicio fisico y ciclo menstrual: Cambios hormonales, psicofisiológicos y sintomatología asociada al ciclo menstrual bumano. Murcia: Secretariado de Publicaciones, Universidad de Murcia. Disponible en: http://hdl.handle.net/10068/292519

Herrera-Gutiérrez, E., Brocal-Pérez, D., Sánchez, D. J. y Rodríguez, J. M. (2012). Relación entre actividad física, depresión y ansiedad en adolescentes. Cuadernos de Psicología del Deporte, 12(Supl. 2), 31-38.

Johnson, E. G., Larsen, A., Ozawa, H., Wilson, C. A. y Kennedy, K. L. (2007). The effects of Pilates-based exercise on dynamic balance in healthy adults. Journal of Bodywork and Movement Therapies, 11, 238-242. Disponible en: http://dx.doi.org/10.1016/j.jbmt.2006.08.008

Kim, J., Singer, R. N., Chung, S., Lee, A., Moon, D. y Kim, W. (2004). Mood alteration related to aerobic, anaerobic and rhythmic exercises. Journal of Human Movement Studies, 47(2), 105-117.

Kull, M. (2003). Physical activity and mental health: Relationships between depressiveness, psychological disorders and physical activity level in women. Biology of Sport, 20(2), 129-138.

Segal, N. A., Hein, J. y Basford, J. R. (2004). The effects of Pilates training of flexibility and body composition: An observational study. Archives of Physical Medicine and Rehabilitation, 85(12), 1977-1981. Disponible en: http://dx.doi.org/10.1016/i.apmr.2004.01.036

Sekendiz, B., Altun, O. Korkusuz, F. y Akin, S. (2007). Effects of Pilates exercise on trunk strength, endurance and flexibility in sedentary adult females. Journal of Bodywork and Movement Therapies, 11, 318-326. Disponible en: http://dx.doi.org/10.1016/i.jbmt.2006.12.002

Sperling, M. y Brum, C. (2006). Who are the people looking for the Pilates method? Journal of Bodywork and Movement Therapies, 10, 328-334. Disponible en: http://dx.doi.org/10.1016/j.jbmt.2005.10.005

Spielberger, C. D., Gorsuch, R. L. y Lushene, R. E. (2002). Cuestionario de Ansiedad Estado-Rasgo (STAI). Madrid: TEA.

Tinoco, M. y Jiménez, M. (2010). Revisión bibliográfica de los estudios de investigación relacionados con el Método Pilates. Scientia, 15(2), 105124.

Tremblay, M. S., Colley, R. C., Saunders, T. J., Healy, G. N. y Owen, N. (2010). Physiological and health implications of a sedentary lifestyle. Applied Physiology, Nutrition and Metabolism, 35, 725-740. Disponible en: http://dx.doi.org/10.1139/H10-079

Tubic, T. y Djordjic, V. (2013). El efecto del ejercicio físico en la salud mental de los niños de edad preescolar. Anales de Psicología, 29(1), 249-256. http://dx.doi.org/10.6018/analesps.29.1.130721

Wankel, L. M. y Berger, B. G. (1990). The psychological and social benefit of sport and physical activity. Journal of Leisure Research, 22, 167-182.

(Articulo recibido: 05-02-2013; revisado: 18-05-2013; aceptado: 25-06-2014) 